\title{
Thermo-catalytic pyrolysis of polystyrene in batch and semi-batch reactors: A comparative study
}

Waste Management \& Research

$1-10$

(c) The Author(s) 2020

Article reuse guidelines:

sagepub.com/journals-permissions DOI: $10.1177 / 0734242 \times 20936746$

journals.sagepub.com/home/wmr

(S)AGE

\author{
Amer Inayat ${ }^{(D)}$, Katerina Klemencova, Barbora Grycova, \\ Barbora Sokolova and Pavel Lestinsky
}

\begin{abstract}
Thermo-catalytic pyrolysis is considered as a promising process for the chemical recycling of waste polymeric materials aiming at converting them into their original monomers or other valuable chemicals. In this regard, process parameters and reactor type can play important roles for an enhanced recovery of the desired products. Polystyrene (PS) wastes are excellent feedstocks for the chemical recycling owing to the capability of PS to be fully recycled. In this respect, the present work deals with the thermo-catalytic pyrolysis of PS in batch and semi-batch reactor setups. The main goal was to perform a comprehensive study on the depolymerisation of PS, thereby investigating the effect of reactor type, catalyst arrangement, feed to catalyst ratio and residence time on the yields of oil and styrene monomer (SM). A further goal was to identify the optimum operating conditions as well as reactor type for an enhanced recovery of oil and SM. It was demonstrated that the semi-batch reactor outperformed the batch reactor in terms of oil and SM yields in both thermal (non-catalytic) and catalytic tests performed at $400^{\circ} \mathrm{C}$. Furthermore, it was shown that the layered arrangement of catalyst (catalyst separated from PS) produced a higher amount of oil with higher selectivity for SM as compared to the mixed arrangement (catalyst mixed with PS). Moreover, the effect of carrier gas flowrate on the product distribution was presented.
\end{abstract}

\section{Keywords}

Pyrolysis, polystyrene, semi-batch reactor, thermo-catalytic process, waste management, chemical recycling

Received 5th March 2020, accepted 31st May 2020 by Editor in Chief Arne Ragossnig.

\section{Introduction}

Over the past five decades, the global plastic production has increased dramatically (Chidambarampadmavathy et al., 2017) and the amount of waste plastics or waste polymeric materials (generated annually) has increased accordingly. In this regard, among the family of thermoplastics, polystyrene (PS) production is invariably at fourth place after polyethylene (PE), polypropylene and polyvinyl chloride (Schwaben, 1999). PS is widely used in packaging, household, construction, electrical and electronics, medical equipment, etc., and consequently a large amount of post-consumer PS waste is generated. Due to poor waste management and low recycling rates of plastic waste globally, a large amount of waste polystyrene (WPS) ends up in landfills as well as in the oceans where it contributes to about $70 \%$ of the plastic debris, which poses a serious threat to marine life (Saido et al., 2012). Furthermore, plastic waste poses long-term risks of contaminating soil and ground water (Hopewell et al., 2009) as landfill is still the main approach used in many countries in handling municipal solid waste that contains WPS and other waste plastics. In Europe, in order to set some basic concepts and definitions related to waste management, in 2008 the European Parliament issued a directive (Directive 2008/98/EC). The directive explains the fivefold waste (management) hierarchy, that is, prevention, preparing for reuse, recycling, energy recovery and disposal. In this directive different goals (to be achieved by 2020) based on the fivefold hierarchy were set (European Parliament and Council, 2008). Consequently, in Europe, during the last 12 years there has been a considerable decrease (about $44 \%$ ) in the amount of post-consumer plastic waste sent to landfill and at the same time the recycling rates have been substantially increased. Nevertheless, $25 \%$ of the plastic post-consumer waste was still sent to landfill in 2018. In order to avoid the abovementioned threats and to achieve a circular economy of plastics, zero landfilling and maximum possible recycling is needed (PlasticsEurope and European Association of Plastics Recycling and Recovery Organisations, 2019).

Plastic recycling processes can mainly be divided into four categories. These include primary (recycling of scrap materials), secondary (recycling through melt extrusion), tertiary (chemical

Institute of Environmental Technology, VSB-Technical University of Ostrava, Ostrava-Poruba, Czech Republic

\section{Corresponding author:}

Amer Inayat, Institute of Environmental Technology, VSB-Technical University of Ostrava, 17. listopadu 15/2172, Ostrava-Poruba, 70800 , Czech Republic.

Email: amer.inayatdvsb.cz 
or feedstock recycling into original monomers or other valuable chemicals) and quaternary (energy recovery through incineration) recycling (Okan et al., 2019; Poulakis and Papaspyrides, 1997). Nowadays chemical or feedstock recycling is attracting much attention as it is environmentally friendly and it can reduce the demand for energy and feedstock and hence can boost the circular economy of plastics (Nzioka et al., 2018; Okan et al., 2019; PlasticsEurope, 2019; Punkkinen et al., 2017). The environmental sustainability of the process can be evaluated through life cycle assessment study.

Owing to the high recyclability of PS (Gu et al., 2010), PS wastes (separated from other wastes) are excellent feedstocks for the chemical recycling for which thermal (non-catalytic) or thermo-catalytic pyrolysis/depolymerisation can be applied. During pyrolysis, polystyrene is cracked in its monomers as well as in dimers and trimers and other compounds such as, toluene (TOL), xylene isomers, methyl styrene, etc. (Kijeńiski and Kaczorek, 2005; Ukei et al., 2000). In addition, depending upon the reaction conditions, degradation of waste polystyrene also produces gaseous products (Miandad et al., 2016; Mo et al., 2014; Shah et al., 2014a; Undri et al., 2014). Thermal pyrolysis is the simplest form of chemical recycling in which carbon-carbon bonds are broken by the application of heat. However, in this case the temperature can be as high as $600^{\circ} \mathrm{C}$ (Ukei et al., 2000). The degradation temperature can be lowered with the use of an appropriate catalyst though. Furthermore, the catalyst can significantly improve the conversion as well as selectivity of the desired products. In this regard, different catalysts have been tested for the thermo-catalytic pyrolysis and reported in the literature. These include solid acids, solid bases and transition metal oxides as well as supported catalysts (metal impregnated on suitable support) (Kijeńiski and Kaczorek, 2005; Ojha and Vinu, 2015; Shah et al., 2017; Ukei et al., 2000). Beside the choice of catalyst, the reactor type or configuration can also play an important role not only on the efficiency of the process but also on the product distribution (Onwudili et al., 2009; Woo et al., 2000). In the literature, for the pyrolysis of plastics, very often batch or semi-batch reactors with either mixed or layered arrangement of catalysts were used (Achilias et al., 2007; Kijeńiski and Kaczorek, 2005; Nisar et al., 2020; Onwudili et al., 2009; Ukei et al., 2000).

According to our literature survey, in recent years, a considerable amount of research has been conducted on thermo-catalytic pyrolysis of waste polymeric materials with a significant part on using PS as feedstock. However, to the best of our knowledge, despite a sizable number of publications in this area, no direct performance comparison of different reactor types (using different catalyst arrangements and residence times) for the degradation of PS has been reported so far. Such comparative study can provide important data that can be used to optimise the process parameters for an enhanced recovery of monomers or other valuable chemicals in the chemical recycling of waste plastics.

Therefore, it is the aim of the present work to perform a comprehensive study on the thermal and thermo-catalytic pyrolysis of PS, thereby investigating the effect of reactor type, catalyst arrangement, feed to catalyst ratio and residence time on the yields of oil and styrene monomer (SM). A further aim is to identify the optimum operating conditions for an enhanced recovery of oil and SM in both batch and semi-batch reactors.

\section{Materials and methods}

\section{Feedstock and catalyst}

Pure PS (3-4 mm diameter spheres) with an average molecular weight of $192000 \mathrm{~g} \mathrm{~mol}^{-1}$ (Sigma Aldrich) was used as model feedstock. The thermogravimetric analysis (TGA) of feedstock was performed in nitrogen $\left(\mathrm{N}_{2}\right)$ atmosphere using a LECO TGA 701 thermogravimetric analyser. For this purpose, $0.45 \mathrm{~g}$ of PS spheres were used. The analysis was carried out using the same temperature program as used for the depolymerisation experiments, that is, heating the sample to $400^{\circ} \mathrm{C}$ with a ramp of $10^{\circ} \mathrm{C}$ min $^{-1}$ with an isothermal step of 1 hour at the final temperature.

For the catalytic depolymerisation of PS, magnesium oxide $(\mathrm{MgO})$ was used as a model catalyst. The $\mathrm{MgO}$ sample was obtained by thermal decomposition of magnesium carbonate $\left(\mathrm{MgCO}_{3}\right)$ powder purchased from Penta Chemicals (Czech Republic). The decomposition of $\mathrm{MgCO}_{3}$ was achieved through calcination at $700^{\circ} \mathrm{C}$ for 5 hours in a Nabertherm muffle furnace of the type P330. In order to confirm the $\mathrm{MgO}$ phase in the calcined sample, X-ray diffraction (XRD) analysis was conducted using a Rigaku SmartLab diffractometer equipped with a D/teX Ultra 250 detector. The textural properties of $\mathrm{MgO}$ were determined by physisorption of nitrogen $\left(\mathrm{N}_{2}\right)$ performed on a Micrometrics 3Flex analyser.

\section{Experimental setup and procedure}

Thermal (non-catalytic) and catalytic pyrolysis experiments were performed in batch and semi-batch reactors at both 400 and $500^{\circ} \mathrm{C}$ and for all runs $4 \mathrm{~g}$ of PS was used as feedstock. For all experiments, a temperature ramp of $10^{\circ} \mathrm{C} \mathrm{min}{ }^{-1}$ was used to reach the target temperature and the experiments were continued for 60 minutes after the target temperature was reached. For the catalytic runs, the $\mathrm{MgO}$ sample obtained was pelletised to get a pellet size in the range $0.63-1 \mathrm{~mm}$.

The batch reactor setup consisted of a muffle oven of the type LMV 2/12 (LAC) equipped with a temperature controller, a round bottom glass tube used as reactor, glass tubing, a condenser with cold water as cooling media and a round bottom flask that was used for collecting the condensate. An additional thermocouple was used to monitor the temperature inside the oven. In order to have inert atmosphere, the setup was purged with $\mathrm{N}$ for 10 minutes at the start of each experiment. The schematic diagram of the test rig is shown in Figure 1 (top). For the thermal runs in batch configuration, a layer of quartz wool was placed on the top of the PS bed which was packed in a round bottom reactor tube (length: $200 \mathrm{~mm}$, diameter: $34 \mathrm{~mm}$ ). Whereas, for the catalytic runs, the catalyst was either mixed with the feedstock (mixed arrangement) 


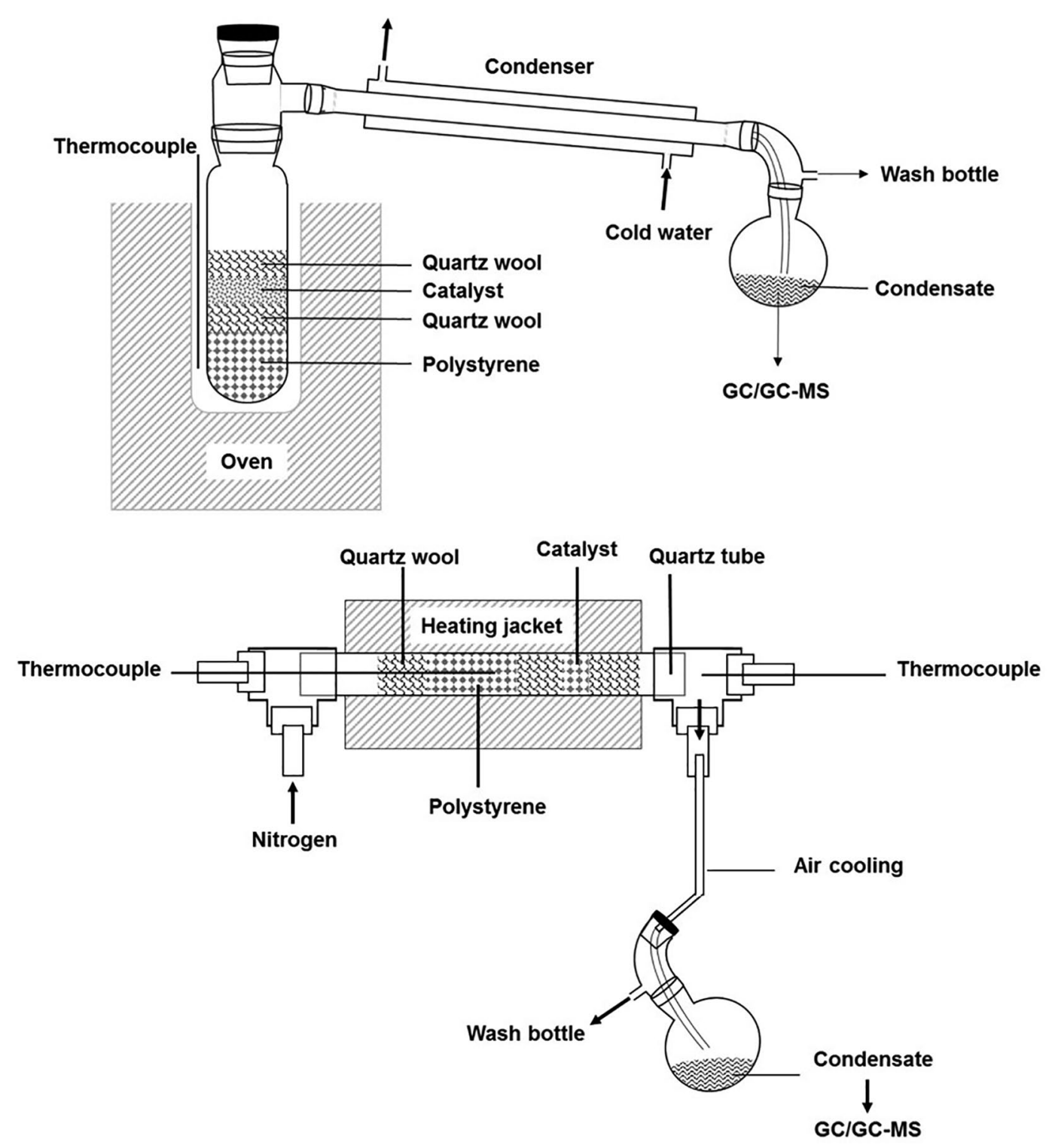

Figure 1. Schematic diagrams of the batch type (top) and semi-batch type (bottom) test rigs, depicting layered arrangement of catalyst.

with a quartz wool layer of about $1 \mathrm{~cm}$ long placed on the top of the mixture or packed between two layers of quartz wool (layered arrangement) placed on the top of PS bed.

For the semi-batch reactor setup, a quartz glass tube of an internal diameter of $17 \mathrm{~mm}$ with a wall thickness of $1.5 \mathrm{~mm}$ and a length of $250 \mathrm{~mm}$ was used as a reactor tube. The tube was heated with a circular heating jacket equipped with a temperature controller. A continuous $\mathrm{N}_{2}$ flow through the reactor tube was provided with the help of a mass flow controller of the type GF17 (Aalborg). In order to avoid styrene condensation inside the Swagelok assembly at the outlet of the reactor tube, an external heating band was used to keep the temperature over $165^{\circ} \mathrm{C}$. The condensation of the products was achieved in a $3 \mathrm{~mm}$ stainless tube through air cooling. The schematic diagram of the test rig is shown in Figure 1 (bottom). For the thermal runs in the semi-batch reactor, the PS bed was packed in the reactor tube using two layers of quartz wool, that is, before and after the bed. For catalytic runs, also in the semi-batch system the catalyst was employed in either mixed arrangement (catalyst mixed with PS beads and the mixture packed between two layers of quartz wool) or layered arrangement (catalyst packed between two layers of quartz wool placed after the PS bed in the flow direction).

\section{Product analysis}

For the batch reactor, the amounts of oil produced and the residue (tar) remaining inside the reactor were determined gravimetrically. For the semi-batch reactor, no residue either in the form of char or tar was observed in any of the experiments performed. Only a small amount of coke on the catalyst surface was observed which was found to be less than $0.5 \mathrm{wt}$. \% based on the feedstock. The amount of oil collected in the condenser flask as well as condensed inside the reactor tube within quartz wool (styrene oligomers; mainly dimers and trimers) near the outlet was determined gravimetrically. The quartz wool loaded with condensed oil was soaked 
and washed in acetone and the oil was collected by subsequent evaporation of the acetone used. The yields of oil, gas and residue were calculated using equations (1)-(3), respectively:

$$
\begin{gathered}
\text { Oil yield }(\text { wt. } \%)=\frac{\text { Mass of oil }}{\text { Mass of } P S} \times 100 \\
\text { Residue yield }(w t . \%)=\frac{\text { Mass of residue }}{\text { Mass of } P S} \times 100 \\
\text { Mass of PS }- \\
\text { Gas yield }(w t . \%)=\frac{(\text { Mass of oil }+ \text { Mass of residue })}{\text { Mass of } P S} \times 100
\end{gathered}
$$

The individual yields of major and minor components of the oil obtained, that is, SM, benzene (BEN), TOL, ethylbenzene (EB), alpha-methylstyrene (AMS) and oligomers were determined with the help of gas chromatography (GC) analysis. For the analysis a YL 6100 GC, equipped with a HP-5 column and a flame ionisation detector was used. The mass fractions of individual oil components were determined by GC analysis. The yields of individual components were then determined by multiplying their mass fractions with the oil yield. In order to identify the range of styrene oligomers, gas chromatography-mass spectrometry (GC-MS) analysis was performed. For this purpose, an Agilent 8890 GC-MS system equipped with a single quad detector 5977B and a HP-5 column was used.

\section{Results and discussion}

\section{Characterisation of the feedstock and catalyst}

The TGA curve in Figure 2 shows that PS started to decompose at around $290.7^{\circ} \mathrm{C}$. By the time the temperature reached $400^{\circ} \mathrm{C}$, it had lost almost $70 \%$ of its mass. The maximum loss occurred at around $399.6^{\circ} \mathrm{C}$. The remaining PS mass was lost within the next 10 minutes during the isothermal step of 1 hour at $400^{\circ} \mathrm{C}$.

The XRD analysis conformed the $\mathrm{MgO}$ phase in the calcined sample (Figure 3). No additional phases were observed (for details, see Supplemental Material). The crystallite size of the $\mathrm{MgO}$ sample was found to be $21 \mathrm{~nm}$ approximately.

The Brunauer-Emmett-Teller (BET) surface area of the $\mathrm{MgO}$ powder sample was found to be $57.6 \mathrm{~m}^{2} \mathrm{~g}^{-1}$. This value is a bit higher as compared to those reported in the literature, for example, 44.4 (Kalogiannis et al., 2018) and $47.4 \mathrm{~m}^{2} \mathrm{~g}^{-1}$ (Ukei et al., 2000). Nevertheless, the BET surface area of the $\mathrm{MgO}$ pellets (used in the catalytic experiments) was found to be $46.8 \mathrm{~m}^{2} \mathrm{~g}^{-1}$, which is comparable to the literature values.

\section{Thermal and catalytic pyrolysis of PS}

In Figure 4, mass spectra showing the product distribution of light (top) and heavy (bottom) oil fractions obtained for the catalytic run at $400^{\circ} \mathrm{C}$ in the semi-batch reactor are presented. All major compounds formed are shown in Figure 4, with monomeric styrene, dimers and trimers dominating the product

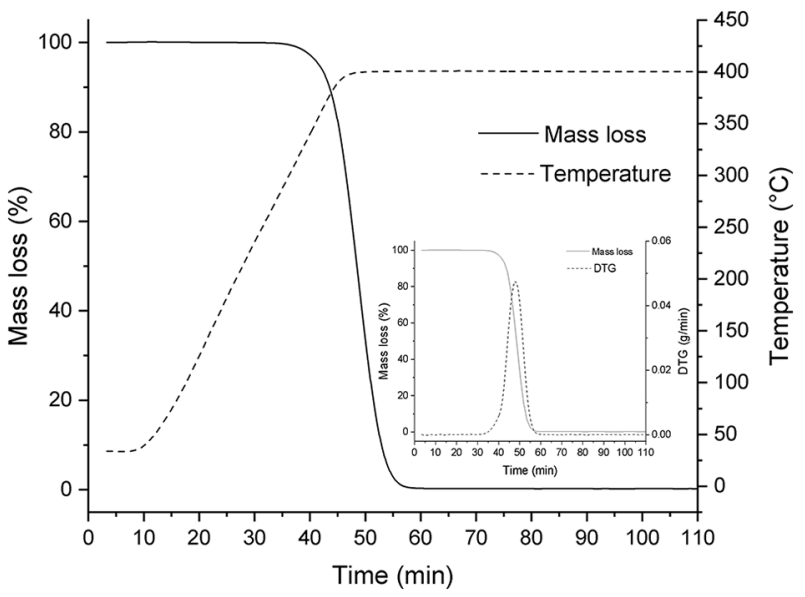

Figure 2. Thermogravimetric analysis curve of polystyrene in nitrogen atmosphere.

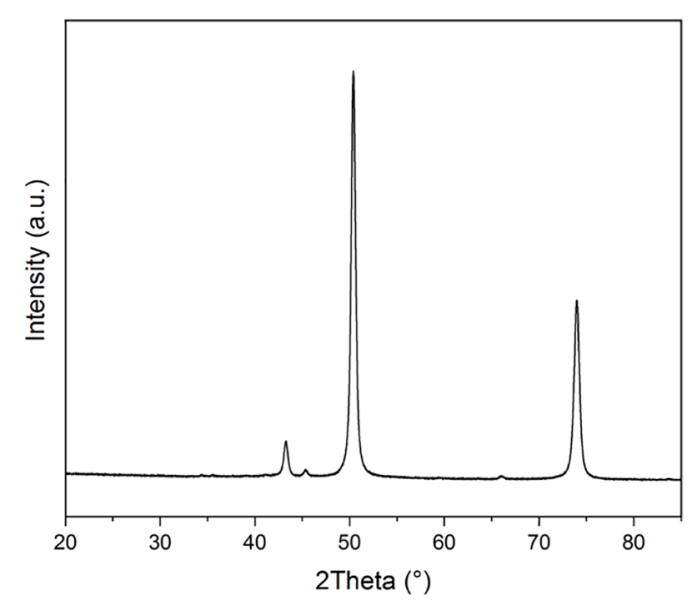

Figure 3. X-ray diffraction pattern of the magnesium oxide sample obtained after calcination.

spectrum (for details, see Supplemental Material). The major compounds as shown in Figure 4 were also reported by other authors (Kijeńiski and Kaczorek, 2005; Nisar et al., 2020; Shah et al., 2014b).

Effect of temperature and reactor configuration. The effect of temperature and reactor type on the yields of oil, residue and gas as well as on the overall yields of the oil components, that is, SM, EB, TOL, AMS and styrene oligomers in both thermal (non-catalytic) and catalytic runs is shown in Figure 5(a) and Figure 5(b). Please note that for all catalytic runs as reported in this subsection, a fixed feed to catalyst ratio of 10 was used and the catalyst pellets were introduced into the reactor in layered arrangement (for details, see the section on experimental setup and procedure). Furthermore, for the semi-batch configuration a constant $\mathrm{N}_{2}$ flow of $50 \mathrm{ml} \mathrm{min}^{-1}$ was used.

From the results shown in Figure 5(a), a trend of higher oil yield for the semi-batch configuration can be observed. In addition, as expected, in both configurations a higher oil yield in the case of catalytic runs was obtained. This is also evident from the SM yield that too increased during the catalysed reactions. The 

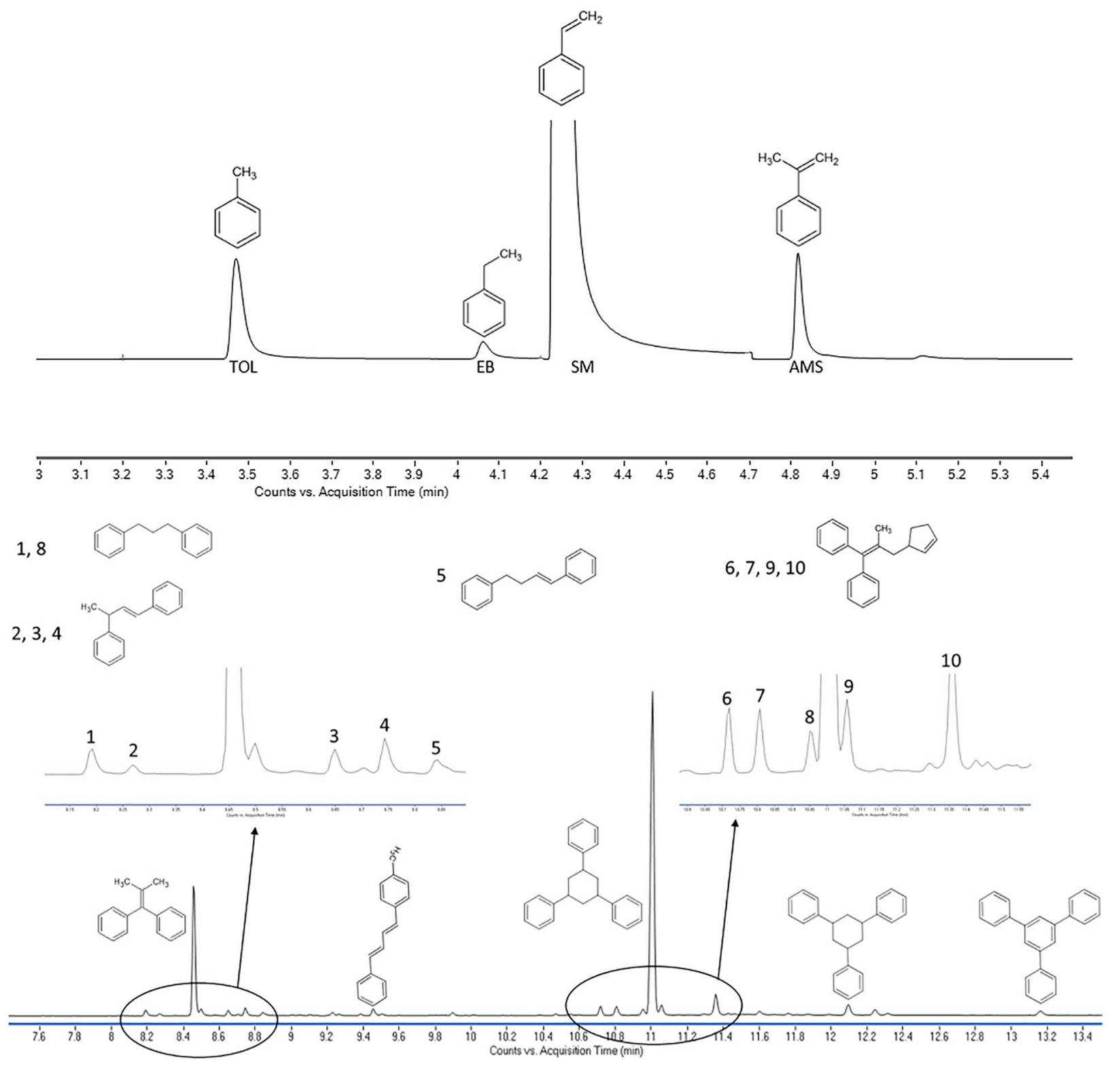

Figure 4. Mass spectra showing product distribution of light (top) and heavy (bottom) oil fractions obtained for the catalytic run at $400^{\circ} \mathrm{C}$ in the semi-batch reactor.

residue production was only observed in the case of batch configuration that decreased with the use of catalyst. The quantity of oligomers (mainly dimers and trimers) was considerably lower in the batch system compared to semi-batch. On the other hand, the amount of gases produced was higher in both thermal and catalytic runs in the batch system. Also, the minor products, that is, TOL, EB and AMS were produced (in somewhat notable amounts) only in the batch reactor, whereas in the semi-batch their contents were a weight percentage of less than 2 . The contents of BEN and xylenes were found to be only in traceable amounts in both systems.

From the results as shown in Figure 5(a), one can observe that the semi-batch configuration fairly outperformed the batch configuration in terms of oil and SM yields in both thermal and catalytic experiments that were performed at $400^{\circ} \mathrm{C}$. This can be attributed to the continuous $\mathrm{N}_{2}$ flow that evacuated the product mixture (including primary volatiles with higher content of SM and oligomers (Zhou et al., 2016) faster than in the batch reactor), thus decreasing the retention time and inhibiting further degradation (Mo et al., 2014; Woo et al., 2000). Longer retention time can lead to secondary reactions producing minor components that can lower the SM content in the condensate. This explains the higher amounts of minor components in the batch system. Also, in this case, the lower amounts of oligomers especially trimers (Table 1) indicate that these compounds (high boiling) could not escape the thermal zone and eventually gave rise to 

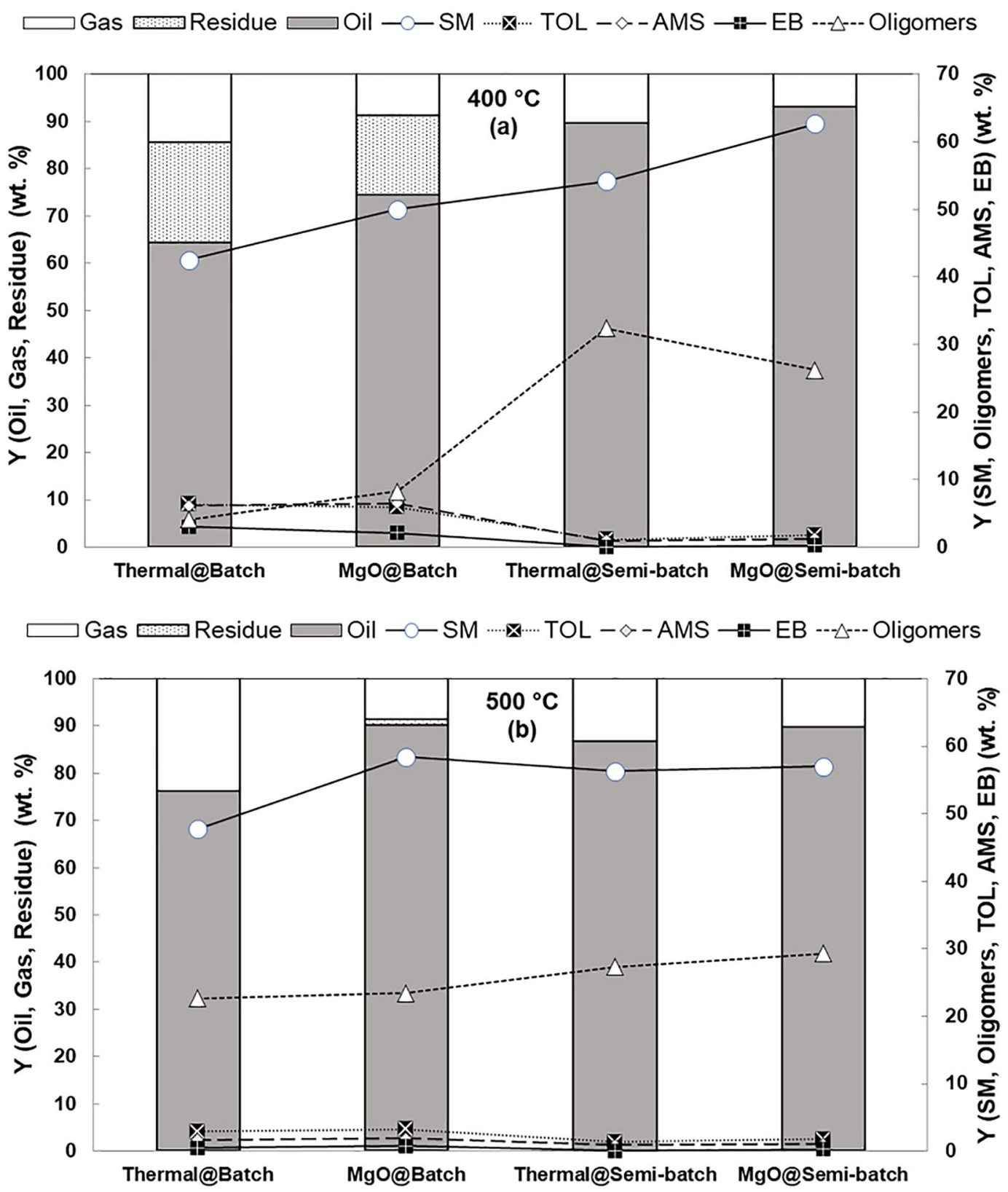

Figure 5. The effect of temperature and reactor configuration.

residue and higher gas production. Thus, the residue produced in the batch system as well as higher amounts of gases and minor components such as, EB, TOL and AMS seem to be the direct consequence of increased retention time of the product mixture. In order to confirm this effect on the product distribution, further experiments in the semi-batch configuration using different $\mathrm{N}_{2}$ flowrates were performed and the results obtained are presented in the subsection on effect of residence time.

In Figure 5(b), the results of similar experiments, as previously discussed, but performed at $500^{\circ} \mathrm{C}$ are shown. It is very interesting to note that for both thermal and catalytic runs, the semi-batch configuration produced very similar results. There was only a slight increase in oil yield for the catalytic run. On the other hand, the catalytic run in the batch system showed considerable increase in the oil as well as SM yield as compared to the thermal run.
The observations at $500^{\circ} \mathrm{C}$ suggest that for the semi-batch configuration, the depolymerisation of PS took place mainly due to the application of heat as the product distribution of thermal as well as catalytic run (at $500^{\circ} \mathrm{C}$ ) is comparable to the thermal run at $400^{\circ} \mathrm{C}$. When comparing the catalytic run at $500^{\circ} \mathrm{C}$ to that performed at $400^{\circ} \mathrm{C}$, the decrease in $\mathrm{SM}$ yield can be explained by the gain in the yield of gaseous products at higher temperature.

Also, for the batch configuration, the decrease in residue content as well as increase in $\mathrm{SM}$ and oils as compared to $400^{\circ} \mathrm{C}$ may be attributed to higher temperature. At $500^{\circ} \mathrm{C}$, most primary volatiles (produced due to thermal depolymerisation) rich in SM and oligomers gained higher kinetic energy (inducing higher local pressure) to escape the reaction zone. This inhibited the secondary reactions that could lead to residue production. In this case, it seems that the higher temperature played a role in evacuating the 
Table 1. Effect of temperature and reactor configuration on the composition of oligomers produced.

\begin{tabular}{|c|c|c|c|c|}
\hline $\begin{array}{l}\text { Temperature } \\
\left({ }^{\circ} \mathrm{C}\right)\end{array}$ & Experiment & $\begin{array}{l}\text { Total oligomers } \\
\text { (weight percentage (wt. \%)) }\end{array}$ & $\begin{array}{l}\text { Dimers } \\
\text { (wt. \%) }\end{array}$ & $\begin{array}{l}\text { Trimers } \\
\text { (wt. \%) }\end{array}$ \\
\hline \multirow[t]{4}{*}{400} & ThermalaBatch & 4.0 & 3.4 & 0.6 \\
\hline & MgOaBatch & 8.3 & 6.5 & 1.8 \\
\hline & ThermalaSemi-batch & 32.4 & 9.5 & 22.9 \\
\hline & MgOaSemi-batch & 26.3 & 11.5 & 14.8 \\
\hline \multirow[t]{4}{*}{500} & ThermalaBatch & 22.7 & 19.0 & 3.7 \\
\hline & MgOaBatch & 23.5 & 19.4 & 4.1 \\
\hline & ThermalaSemi-batch & 27.3 & 11.5 & 15.8 \\
\hline & MgOaSemi-batch & 29.3 & 18.6 & 10.7 \\
\hline
\end{tabular}

primary volatiles. Furthermore, in the catalytic run the oil and SM yield is further enhanced due to the catalytic effect, and the performance reached by the batch system even slightly surpassed the one of the semi-batch system. Here, a slightly worse performance of the semi-batch reactor might suggest that the $\mathrm{N}_{2}$ flowrate (that was kept constant at 400 and $500^{\circ} \mathrm{C}$ ) was too high at $500^{\circ} \mathrm{C}$.

Effect of catalyst arrangement. In this subsection, the results of catalytic experiments performed at 400 and $500^{\circ} \mathrm{C}$ in both batch and semi-batch reactors using different catalyst arrangements are presented. For all experiments, a feed to catalyst ratio of 10 was used and for the semi-batch system the $\mathrm{N}_{2}$ flowrate was kept at $50 \mathrm{ml} \mathrm{min}{ }^{-1}$. The catalyst pellets were either mixed with PS beads or placed as a single layer between two layers of quartz wool (for the details, please see the section on experimental setup and procedure).

In Figure 6(a), the higher oil yields for layered arrangements in both reactors suggest that the catalytic depolymerisation of PS mainly took place in the gas phase. This means that the catalytic depolymerisation of PS involved the degradation of vapourised fragments of PS chains (Ukei et al., 2000), where vapourised fragments were mainly formed during the thermal degradation. The vapourised fragments started to depolymerise already before entering the catalyst bed (in layered arrangement) which then added its effect to the already initiated depolymerisation phenomenon under the influence of heat. In this case, the maximum amount of vapourised fragments/reaction mixture had a chance to encounter the catalyst pellets while leaving the thermal zone as compared to the mixed arrangement. This increased the overall catalytic effect resulting in higher amounts of oil in layered arrangement. On the other hand, the performance of the mixed catalyst arrangement in terms of oil yield approached to that of the thermal (non-catalytic) run at $400^{\circ} \mathrm{C}$, indicating the decreased effectivity of the catalyst in this arrangement.

The catalytic tests with layered and mixed arrangements were also performed at $500^{\circ} \mathrm{C}$ and the results obtained are shown in Figure 6(b). From these results, it seems that at $500^{\circ} \mathrm{C}$ the catalyst arrangement influenced the oil or SM yield only slightly. Also, the evacuation of reaction products with a carrier gas (with a flowrate as high as of $50 \mathrm{ml} \mathrm{min}^{-1}$ ) had a slightly adverse effect (as discussed in the subsection on effect of temperature and reactor configuration) on oil or SM yield. This may be due to decrease in the necessary residence time (contact time with the catalyst) required for the maximum production of oil or SM. This means that although the semi-batch system performed better at $400^{\circ} \mathrm{C}$ compared to the batch system, at higher temperature an optimum flowrate of the carrier gas must be found and set in order to take full advantage of the layered arrangement as well as the semi-batch configuration. At higher temperatures (as described earlier) the product mixture will gain higher kinetic energy and the tendency of leaving the reactor faster. This explains the higher amounts of dimers in the condensate at $500^{\circ} \mathrm{C}$ as compared to $400^{\circ} \mathrm{C}$ (see Table S1 in Supplemental Material). The comparatively lower amounts of trimers $\left(\right.$ at $500^{\circ} \mathrm{C}$ ) on the other hand can be attributed to their further transformation into dimers at higher temperature.

Effect of residence time. In order to investigate the effect of residence time on the product distribution, experiments with different flowrates of the carrier gas were carried out. For these experiments, $400^{\circ} \mathrm{C}$ was chosen as the reaction temperature because at $500^{\circ} \mathrm{C}$ the carrier gas had a small effect on the oil or $\mathrm{SM}$ yields (see previous sections). For all experiments in this subsection, a feed to catalyst ratio of 10 was used. The results obtained are shown in Figure 7.

In Figure 7, it can be seen that the batch reactor (no carrier gas) gave the lowest amount of oil and SM. As already discussed, at $400^{\circ} \mathrm{C}$ the semi-batch configuration with a $\mathrm{N}_{2}$ flowrate of $50 \mathrm{ml}$ $\mathrm{min}^{-1}$ gave higher yields of oil and SM as compared to the batch system. This trend was kept even at lower $\mathrm{N}_{2}$ flowrate. Although, the yields of oil and SM were lower than that at $50 \mathrm{ml} \mathrm{min}^{-1}$. In addition, it is interesting to see that at very low or very high flowrates of the carrier gas, the performance of the semi-batch system in terms of SM or oil yield tends to approach that of the batch system.

These observations further confirm that indeed at $400^{\circ} \mathrm{C}$ the product distribution is greatly affected by the flowrate of the carrier gas, in other words the residence time of the primary volatiles. Without a carrier gas, a part of volatile products unable to leave the reaction zone went under further degradation to produce minor compounds and residue. On the other hand, very high flowrates might have led to an inadequate (low) residence time resulting in incomplete reaction leaving more oligomers in the products. The results in Figure 7 show a similar trend of first increase and then decrease in the yields of oil and SM (with increasing flowrates) as compared to the ones reported by Mo 

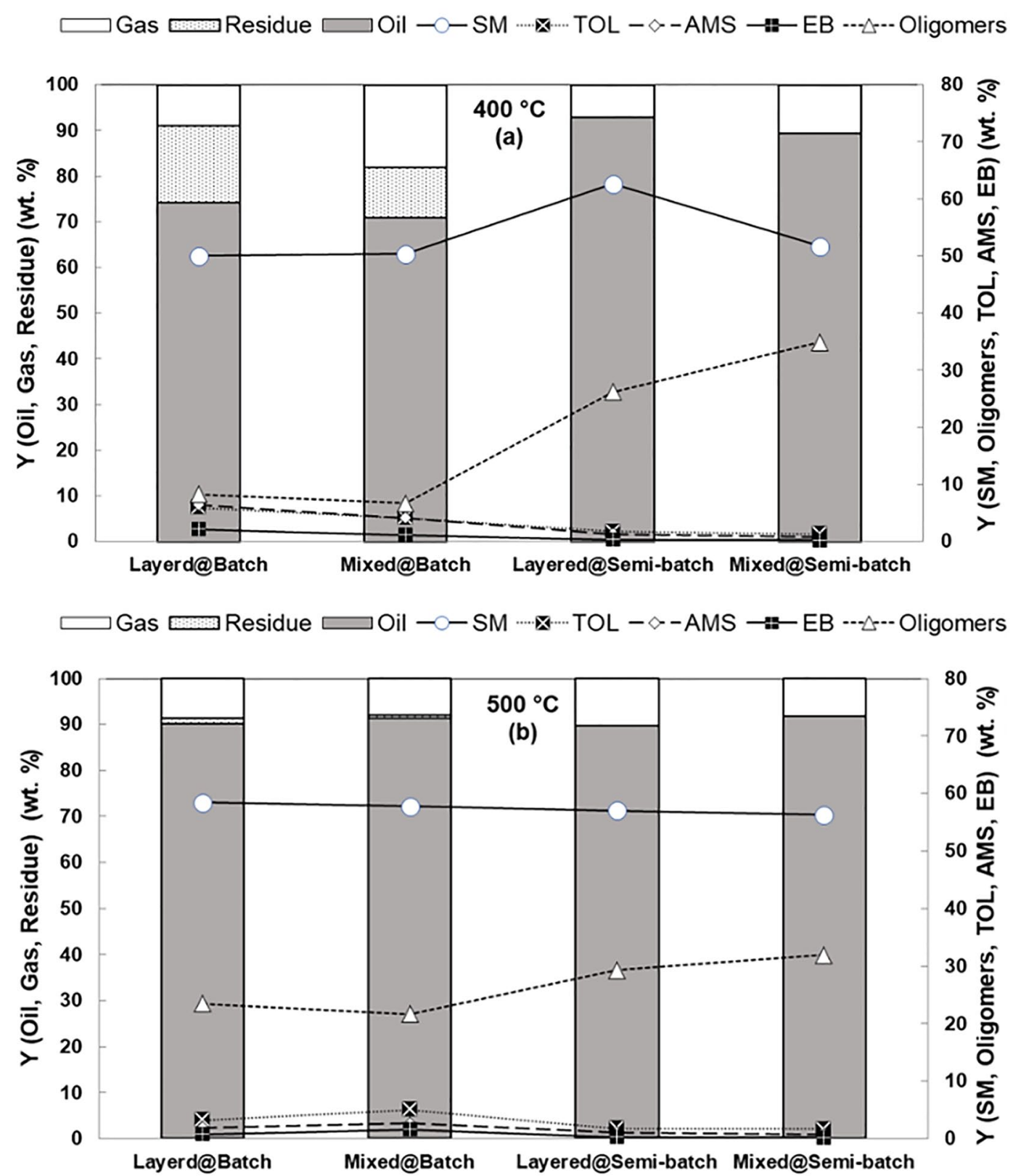

Figure 6. The effect of catalyst arrangement.

et al. (2014), who showed that flowrate of the carrier gas had a quadratic effect on the styrene yield. Mo et al. studied thermal degradation in a semi batch reactor, however, with different reactor dimensions and the range of carrier gas flowrates.

For the present study, the flowrate of $50 \mathrm{ml} \mathrm{min}^{-1}$ seems to be the optimum flowrate of the carrier gas for enhanced oil and SM recovery at $400^{\circ} \mathrm{C}$.

Effect of feed to catalyst ratio. For the present study, three experiments with different feed to catalyst ratios were carried out. The experiments were performed in the semi-batch reactor at $400^{\circ} \mathrm{C}$ with $\mathrm{N}_{2}$ flowrate of $50 \mathrm{ml} \mathrm{min}^{-1}$. The results are depicted in Figure 8.

The results show that a lower feed to catalyst ratio (higher amount of catalyst) produced a higher amount of oil. Although, the oil yield with the feed to catalyst ratio of 5 differs only slightly when compared to the one obtained at the ratio of 10 . Furthermore, this increase came at the cost of decrease in SM content in the product oil which, however, showed higher content of oligomers. In addition, an interesting trend of first increase and then decrease in SM content with increasing feed to catalyst ratio can be observed which is reversed for the oligomers.

These results suggest that with a lower amount of catalyst, the catalytic degradation tends to approach the thermal degradation in terms of oil and SM content. However, with a higher amount of catalyst the decrease in SM content was unexpected. In the literature a similar effect was reported (Aljabri et al., 2017) but in that case a lower-reaction temperature was used. Aljabri et al. explained this effect on the basis of partial repolymerisation of SM to form oligomers over the catalyst surface. 


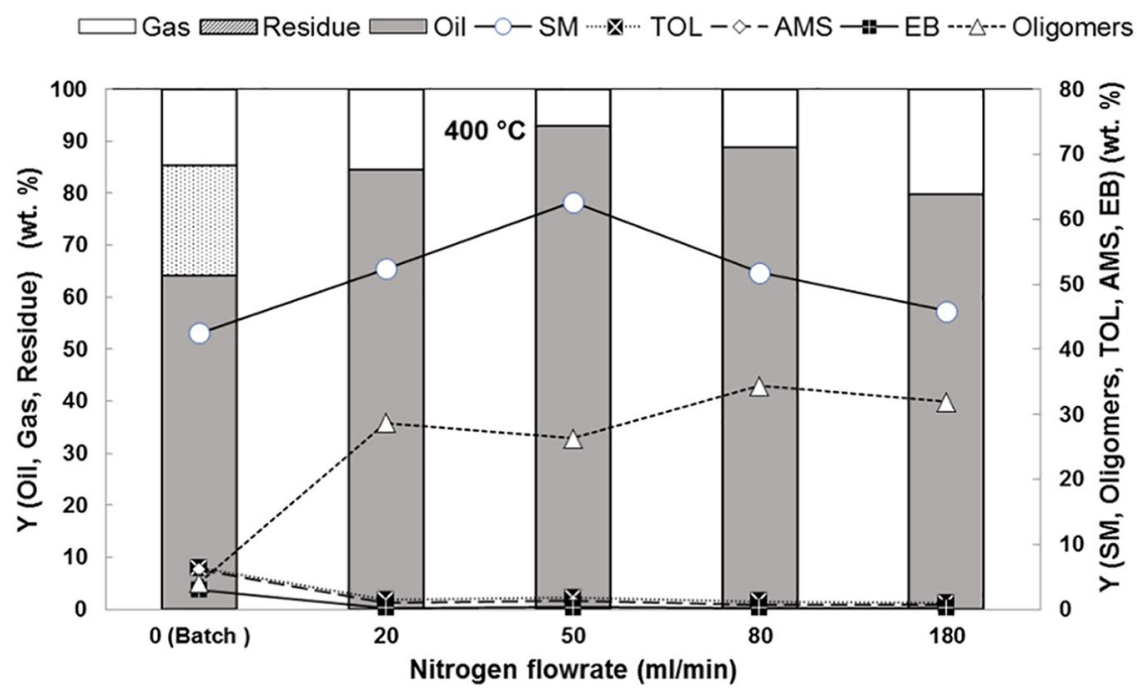

Figure 7. The effect of residence time.

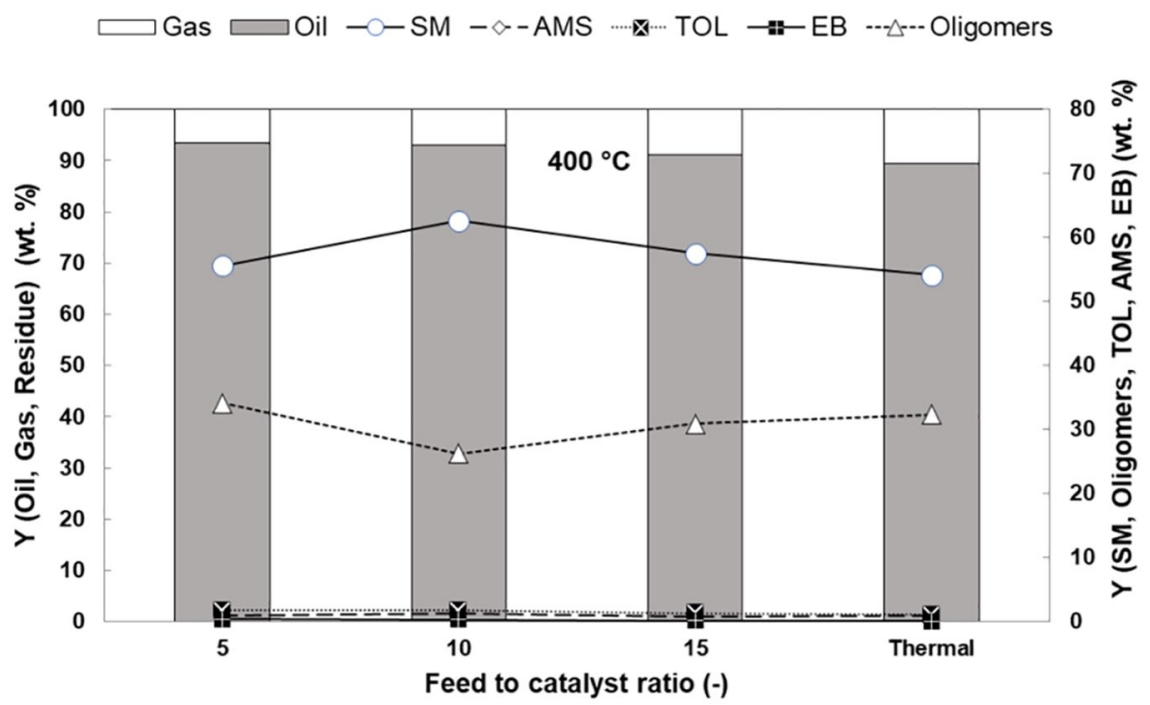

Figure 8. The effect of feed to catalyst ratio for layered arrangement.

\section{Conclusion}

The presence of plastic wastes in the oceans as well as in landfills presents serious risks to human and animal health and for the environment. Promoting recycling of waste plastic materials can not only reduce these risks but also boost the circular economy of plastics. Here, chemical or feedstock recycling of waste plastics can play a key role which can be achieved through thermal or catalytic pyrolysis/depolymerisation. The present work was devoted to investigations on the thermal and catalytic pyrolysis of PS. The main aim was to study the effect of reactor type and operating conditions on the yields of oil and SM. A further aim was to identify the optimum operating conditions as well reactor configuration for an enhanced recovery of oil and styrene in thermal and catalytic pyrolysis of PS.

It was demonstrated that the layered arrangement of catalyst (feedstock and catalyst separated with quartz wool) not only produced a higher amount of oil but also gave higher selectivity for
SM, indicating that the catalytic degradation of PS took place mainly in the gas phase. Furthermore, it was shown that at $400^{\circ} \mathrm{C}$, the semi-batch reactor configuration outperformed the batch system in both thermal and catalytic tests, which exemplifies the positive effect of forced evacuation of the products. At temperature as high as $500^{\circ} \mathrm{C}$, the product distribution was rather independent of the reactor configuration or catalyst arrangement, either mixed or layered. Moreover, it was shown that in the case of semi-batch system, the maximum oil and styrene yields were obtained when the feed to catalyst ratio of 10 was used and the flowrate of the carrier gas was set to give a residence time of 19 seconds. The data obtained from the present study can be used to optimise the process for thermocatalytic pyrolysis of waste plastics.

\section{Declaration of conflicting interests}

The authors declared no potential conflicts of interest with respect to the research, authorship, and/or publication of this article. 


\section{Funding}

The authors disclosed receipt of the following financial support for the research, authorship, and/or publication of this article: The work was supported from ERDF "Institute of Environmental Technology - Excellent Research" (Grant Number: CZ.02.1.01/0. 0/0.0/16 019/0000853). Experimental results were accomplished by using Large Research Infrastructure - Energy Waste Recovery and Gas Treatment supported by the Ministry of Education, Youth and Sports of the Czech Republic under Project Number LM2018098.

\section{ORCID iD}

Amer Inayat iD https://orcid.org/0000-0001-7457-6430

\section{Supplemental material}

Supplemental material for this article is available online.

\section{References}

Achilias DS, Kanellopoulou I, Megalokonomos P, et al. (2007) Chemical recycling of polystyrene by pyrolysis: Potential use of the liquid product for the reproduction of polymer. Macromolecular Materials and Engineering 292: 923-934.

Aljabri NM, Lai Z, Hadjichristidis N, et al. (2017) Renewable aromatics from the degradation of polystyrene under mild conditions. Journal of Saudi Chemical Society 21: 983-989.

Chidambarampadmavathy K, Karthikeyan OP and Heimann K (2017) Sustainable bio-plastic production through landfill methane recycling. Renewable and Sustainable Energy Reviews 71: 555-562.

European Parliament and Council(2008) Directive 2008/98/EC of the European Parliament and of the Council of 19 November 2008 on waste and repealing certain directives (Text with EEA relevance). Available at: https:// eur-lex.europa.eu/legal-content/EN/TXT/?uri=celex\%3A32008L0098 (accessed 18 June 2020).

Gu R, Lee O and Salehzadah Y (2010) An Investigation into Polystyrene Recycling at UBC. Available at: https://www.sustain.ubc.ca/sites/sustain.ubc.ca/files/seedslibrary/APSC262_Styrofoam_Group3_CLEAN FINAL.pdf (accessed 18 June 2020).

Hopewell J, Dvorak R and Kosior E (2009) Plastics recycling: Challenges and opportunities. Philosophical Transactions of the Royal Society B: Biological Sciences 364: 2115-2126.

Kalogiannis KG, Stefanidis SD, Karakoulia SA, et al. (2018) First pilot scale study of basic vs acidic catalysts in biomass pyrolysis: Deoxygenation mechanisms and catalyst deactivation. Applied Catalysis B: Environmental 238: 346-357.

Kijeńiski J and Kaczorek T (2005) Catalytic degradation of polystyrene. Polimery 50: 60-63.

Miandad R, Nizami AS, Rehan M, et al. (2016) Influence of temperature and reaction time on the conversion of polystyrene waste to pyrolysis liquid oil. Waste Management 58: 250-259.

Mo Y, Zhao L, Wang Z, et al. (2014) Enhanced styrene recovery from waste polystyrene pyrolysis using response surface methodology coupled with Box-Behnken design. Waste Management 34: 763-769.

Nisar J, Ali G, Shah A, et al. (2020) Pyrolysis of polystyrene waste for recovery of combustible hydrocarbons using copper oxide as catalyst. Waste
Management \& Research 0734242X2090440. Epub before print 20 February 2020. DOI: 10.1177/0734242X20904403.

Nzioka AM, Yan CZ, Kim MG, et al. (2018) Improvement of the chemical recycling process of waste carbon fibre reinforced plastics using a mechanochemical process: Influence of process parameters. Waste Management \& Research 36: 952-964.

Ojha DK and Vinu R (2015) Resource recovery via catalytic fast pyrolysis of polystyrene using zeolites. Journal of Analytical and Applied Pyrolysis 113: 349-359.

Okan M, Aydin HM and Barsbay M (2019) Current approaches to waste polymer utilization and minimization: A review. Journal of Chemical Technology \& Biotechnology 94: 8-21.

Onwudili JA, Insura N and Williams PT (2009) Composition of products from the pyrolysis of polyethylene and polystyrene in a closed batch reactor: Effects of temperature and residence time. Journal of Analytical and Applied Pyrolysis 86: 293-303.

PlasticsEurope (2019) The Circular Economy for Plastics - A European Overview 1-9. Available at: https://www.plasticseurope.org/en/resources/ publications/1899-circular-economy-plastics-european-overview (accessed 18 June 2020).

PlasticsEurope and European Association of Plastics Recycling and Recovery Organisations (2019) Plastics - The Facts 2019. 14 October 2019. Available at: https://www.plasticseurope.org/application/files/9715/7129/9584/FINAL_web_version_Plastics_the facts2019 14102019.pdf (accessed 18 June 2020).

Poulakis JG and Papaspyrides CD (1997) Recycling of polypropylene by the dissolution/reprecipitation technique: I. A model study. Resources, Conservation and Recycling 20: 31-41.

Punkkinen H, Oasmaa A, Laatikainen-Luntama J, et al. (2017) Thermal conversion of plastic-containing waste: A review. Research Report No D4.1-22 Helsinki 2017. Finland: CLIC. Available at: http://arvifinalreport.fi/files/Thermal $\% 20$ conversion $\% 20$ of $\% 20$ plastic-containing $\% 20$ waste $\% 20 \mathrm{~A} \% 20$ review.pdf (accessed 18 June 2020).

Saido K, Amamiya K, Sato H, et al. (2012) Analysis of styrene oligomer contaminants generated from marine debris polystyrene on the coast of Okinawa. Bunseki Kagaku 61: 629-636.

Schwaben HD (1999) Polystyrene (PS). Kunststoffe Plast Europe 89: 14-16.

Shah J, Jan MR and Adnan (2014a) Catalytic activity of metal impregnated catalysts for degradation of waste polystyrene. Journal of Industrial and Engineering Chemistry 20: 3604-3611.

Shah J, Jan MR and Adnan (2014b) Conversion of waste polystyrene through catalytic degradation into valuable products. Korean Journal of Chemical Engineering 31: 1389-1398.

Shah J, Jan MR and Adnan (2017) Metal decorated montmorillonite as a catalyst for the degradation of polystyrene. Journal of the Taiwan Institute of Chemical Engineers 80: 391-398.

Ukei H, Hirose T, Horikawa S, et al. (2000) Catalytic degradation of polystyrene into styrene and a design of recyclable polystyrene with dispersed catalysts. Catalysis Today 62: 67-75.

Undri A, Frediani M, Rosi L, et al. (2014) Reverse polymerization of waste polystyrene through microwave assisted pyrolysis. Journal of Analytical and Applied Pyrolysis 105: 35-42.

Woo OS, Ayala N and Broadbelt LJ (2000) Mechanistic interpretation of basecatalyzed depolymerization of polystyrene. Catalysis Today 55: 161-171.

Zhou J, Qiao Y, Wang W, et al. (2016) Formation of styrene monomer, dimer and trimer in the primary volatiles produced from polystyrene pyrolysis in a wire-mesh reactor. Fuel 182: 333-339. 